\section{ORIGINAL RESEARCH}
S. Partovi
B. Jacobi
N. Rapps
L. Zipp
S. Karimi
F. Rengier
J.K. Lyo
C. Stippich

\title{
Clinical Standardized fMRI Reveals Altered Language Lateralization in Patients with Brain Tumor
}

\begin{abstract}
BACKGROUND AND PURPOSE: Brain tumors affecting language-relevant areas may influence language lateralization. The purpose of this study was to systematically investigate language lateralization in brain tumor patients using clinical language fMRI, comparing the results with a group of healthy volunteers.
\end{abstract}

\begin{abstract}
MATERIALS AND METHODS: Fifty-seven strictly right-handed patients with left-hemispheric-space intracranial masses (mainly neoplastic) affecting either the Broca area $(n=19)$ or Wernicke area $(n=38)$ were prospectively enrolled in this study. Fourteen healthy volunteers served as a control group. Standardized clinical language fMRI, using visually triggered sentence- and word-generation paradigms, was performed on a 1.5T MR scanner. Semiautomated analyses of all functional data were conducted on an individual basis using BrainVoyager. A regional lateralization index was calculated for Broca and Wernicke areas separately versus their corresponding right-hemisphere homologs.
\end{abstract}

RESULTS: In masses affecting the Broca area, a significant decrease in the lateralization index was found when performing word generation ( $P=.0017)$, whereas when applying sentence generation, the decrease did not reach statistical significance $(P=.851)$. Masses affecting the Wernicke area induced a significant decrease of the lateralization index when performing sentence generation $(P=$ .0007), whereas when applying word generation, the decrease was not statistically significant $(P=$ .310).

CONCLUSIONS: Clinical language fMRI was feasible for patients with brain tumors and provided relevant presurgical information by localizing essential language areas and determining language dominance. A significant effect of the brain masses on language lateralization was observed, with a shift toward the contralesional, nondominant hemisphere. This may reflect compensatory mechanisms of the brain to maintain communicative abilities.

ABBREVIATIONS: BOLD = blood oxygen level-dependent; $\mathrm{BR}=$ Broca right; $\mathrm{HRF}=$ hemodynamic response function; $\mathrm{LI}=$ lateralization index; $\mathrm{SG}=$ sentence generation; $\mathrm{WG}=$ word generation; $\mathrm{WHO}=$ World Health Organization; WR $=$ Wernicke right

A lteration of language lateralization may occur in patients with intracranial space-occupying masses affecting the dominant hemisphere and may be a sign of the brain's plasticity to compensate for possible language deficits. The probability of such alterations will increase when the brain lesions infiltrate or displace eloquent language areas, namely, the Broca area (anatomic homolog is the inferior frontal gyrus) and Wernicke area (anatomic homologs are the superior temporal gyrus, supramarginal gyrus, and angular gyrus). In contrast

Received January 3, 2012; accepted after revision March 5, 2012.

From the Department of Neuroradiology (S.P., L.Z., F.R., C.S.), University Hospital of Basel, Basel, Switzerland; Department of Radiology, Section of Neuroradiology (S.P., S.K., J.K.L.), Memorial Sloan-Kettering Cancer Center, New York, New York; Clinical Cooperation Unit Molecular Hematology/Oncology (B.J.), German Cancer Research Center, Heidelberg, Germany; Department of Psychosomatic Medicine and Psychotherapy (N.R.), University Hospital of Tuebingen, Tuebingen, Germany; Departments of Internal Medicine V (B.J.), Neuroradiology (N.R., C.S.), and Diagnostic and Interventional Radiology (F.R.), University Hospital of Heidelberg, Heidelberg, Germany.

Paper previously presented as an oral presentation at the 97th Scientific Assembly and Annual Meeting of the Radiologic Society of North America, November 28, 2011; Chicago, Illinois. It was selected for the RSNA press kit, which was distributed by the RSNA Public Information and Media Relations in association with the Annual Meeting of the Radiologic Society of North America 2011.

S.P. and B.J. contributed equally to this work.

Please address correspondence to Sasan Partovi, University Hospital of Basel, Department of Neuroradiology, Petersgraben 4, 4031 Basel, Switzerland; e-mail: sasanp@gmx.de http://dx.doi.org/10.3174/ajnr.A3137 to motor function, there are no reliable landmarks available on structural images of the human brain to localize essential language areas, giving functional neuroimaging an important diagnostic role when planning and performing safe and function-preserving surgical interventions in areas that are relevant for language. ${ }^{1}$ The same holds true for determining the language-dominant hemisphere before neurosurgery. ${ }^{2}$

BOLD fMRI is a valuable neuroimaging tool for assessing language functions noninvasively, namely, for localizing essential language areas and thereby assessing their spatial relationship to brain tumors, and for determining the languagedominant hemisphere, by analyzing language lateralization. ${ }^{3-8}$ fMRI language lateralization also has the potential to allow for the study of brain plasticity in different pathologic states, such as epilepsy and stroke. ${ }^{9-15}$

The mechanisms underlying neuronal plasticity induced by brain lesions are largely unknown. Changes in transcallosal and collateral inhibition of relevant brain areas for language processing have been suggested as underlying mechanisms. Others have discussed modification of synaptic activity and unmasking of latent horizontal connections. ${ }^{16,17}$ The type, extent, and timeframe of brain plasticity mechanisms may also depend on the nature of brain lesions and their spatiotemporal attitudes.

A few studies have investigated postsurgical brain plasticity 
in patients with brain tumors by performing fMRI, focusing predominantly on the motor cortex. ${ }^{18-21}$ To our knowledge, no fMRI language investigation exists that analyzes language lateralization in a larger and clinically representative cohort of patients with brain tumors affecting the Broca and Wernicke areas. However, brain plasticity, as expressed by alterations in language lateralization, seems to play a major role in compensating for speech deficits in the case of long-standing and growing lesions, such as brain tumors, whereas adaptation does not occur within a short timeframe, such as in the case of a single catastrophic case of brain damage, like massive stroke. $^{22-24}$ These alterations in cortical language networks can be investigated noninvasively using clinical fMRI, through determination of the dominant language hemisphere by calculating lateralization indices for the corresponding languagerelevant brain areas. ${ }^{25-28}$ Compared with the invasive Wada test for assessing language lateralization, fMRI demonstrated high sensitivity and specificity in a meta-analysis. ${ }^{2}$ Previous research has found 2 different types of cortical reorganization as a response to a brain lesion: ipsilateral perilesional cortical reorganization or a shift of the dominant hemisphere to the contralateral side.

In light of the latter observation, the purpose of this study was to systematically analyze presurgical fMRI language lateralization in a large cohort of strictly right-handed patients with left hemispheric brain tumors either affecting the Broca language area or Wernicke language area, and to compare language lateralization in patients with reference data obtained in healthy volunteers. With this study, we aimed to assess the effects of space-occupying brain masses on BOLD fMRI language lateralization in a typical clinical setting.

The clinical standard fMRI protocol included 2 language paradigms: SG and WG, which have been previously established in healthy volunteers and validated against invasive reference procedures in patients with brain tumor. ${ }^{25,29}$

\section{Materials and Methods}

The study was approved by the local ethics commission and was in line with the Helsinki Declaration of Human Rights. All patients participated in the study after written informed consent.

\section{Study Population}

Over a period of 4 years, all consecutive patients with brain tumors at the University Hospital of Heidelberg were investigated in a prospective study design with conventional MR imaging. If a spaceoccupying mass was detected within a critical distance of anatomy homologs of Broca (inferior frontal gyrus) or Wernicke (superior temporal gyrus, supramarginal gyrus, angular gyrus) areas in the left hemisphere, language fMRI was performed. Critical distance was defined as tumor-related T1-signal alteration, with or without contrast enhancement, in the following anatomic structures: the left inferior frontal gyrus, the dorsal aspect of the left superior temporal gyrus, the left supramarginal gyrus, and/or the adjacent angular gyrus. In extraaxial masses, direct displacement and/or compression of these anatomic structures by the tumor were considered as critical.

Handedness was assessed in each patient through a modified questionnaire, following that outlined by Annett, ${ }^{30}$ and only strictly right-handed patients were included in this study. Fifty-seven patients were enrolled in this fMRI study ( 32 men and 25 women; mean age, 44.3 years). Among these 57 patients, 38 patients had space-

\begin{tabular}{lc}
\hline $\begin{array}{l}\text { Baseline characteristics of the control (healthy volunteers) and } \\
\text { patient groups }\end{array}$ \\
\hline Healthy volunteers $(n=14)$ & $26 \pm 5$ \\
Age, years ( \pm SD) & $7(50 \%)$ \\
Male sex ( $[\%])$ & \\
Patient population ( $n=57)$ & $44 \pm 16$ \\
Age, years ( \pm SD) & $32(56 \%)$ \\
Male sex ( $[$ [\%]) & \\
Tumor localization ( $n[\%])$ & $19(33 \%)$ \\
Affecting Broca area & $38(67 \%)$ \\
Affecting Wernicke area & \\
WHO tumor grade ( $n[\%])$ & $9(16 \%)$ \\
I & $12(21 \%)$ \\
II & $4(4 \%)$ \\
III & $12(21 \%)$ \\
IV & $16(28 \%)$ \\
No WHO grade & $4(7 \%)$ \\
No histology available & \\
\hline Note:-For tumor data, $n=57$. &
\end{tabular}

Note:-For tumor data, $n=57$.

occupying masses (28 neoplastic and 10 non-neoplastic) affecting the Wernicke area and 19 had space-occupying masses ( 17 neoplastic and 2 non-neoplastic) affecting the Broca area. The histologically confirmed diagnoses included 11 cases of oligodendroglioma (3 affecting Wernicke area and 8 affecting Broca area), 12 cases of low-grade glioma ( 7 affecting Wernicke area and 5 affecting Broca area), 13 cases of high-grade glioma (11 affecting Wernicke area and 2 affecting Broca area), 6 cases of cavernoma (5 affecting Wernicke area and 1 affecting Broca area), 4 metastases (3 affecting Wernicke area and 1 affecting Broca area), 4 tumors with unknown entity ( 3 affecting Wernicke area and 1 affecting Broca area), 2 meningiomas (as extra-axial tumors, both affecting Wernicke area), 1 abscess (affecting Wernicke area), 1 cortical dysplasia (affecting Wernicke area), 1 gliosarcoma (affecting Broca area), 1 malignant pleomorphic cellular tumor (affecting Wernicke area), and 1 nonspecific glial proliferation (affecting Wernicke area). Clinically, 23 of the 57 patients suffered from aphasic symptoms, but none of these patients were globally aphasic. Each patient enrolled was able to perform the fMRI paradigms. Fourteen healthy right-handed volunteers $(7 \mathrm{men}$ and 7 women) between 21 and 47 years of age (mean age, 27 years) served as a control group for this study, as published previously. ${ }^{31}$ Before language fMRI, volunteers underwent structural MR imaging without contrast administration to exclude occult neurologic disease. Baseline characteristics of the patients and volunteers are provided in the Table.

\section{Functional and Morphologic MR Imaging}

Functional and morphologic whole-brain MR imaging was performed using the same 1.5T MR scanner (Marconi EDGE; Cleveland, Ohio) in all patients, using a conventional head coil. Movement artifacts during functional and conventional MR imaging were minimized by relaxed positioning of the extremities and by fixing the head with preformed foam cushions, with sparing of the temporomandibular joint. For BOLD fMRI, a T2*-weighted single-shot echoplanar imaging sequence $(\mathrm{TR}=3.0$ seconds, $\mathrm{TE}=80 \mathrm{~ms}$, FOV $=$ $256 \times 256 \mathrm{~mm}^{2}$, matrix $=128 \times 128$ voxels, pulse angle $\left.=90^{\circ}\right)$ was approved. Twenty-two transverse sections (section thickness $5 \mathrm{~mm}$ and section distance $1 \mathrm{~mm}$ ) were acquired for whole-head coverage. With the head fixed in the exact same position, a $\mathrm{T} 1^{\star}$-weighted anatomic 3D MR imaging (radio-frequency spoiled fast low-angle shot sequence, with $\mathrm{TR}=30 \mathrm{msec}$ and $\mathrm{TE}=4.4 \mathrm{~ms}$ ) was acquired for 
superimposition on fMRI, with 135 transverse sections and section thickness of $1.3 \mathrm{~mm}$ in patients undergoing neuronavigation, and 120 sagittal sections and section thickness of $1.5 \mathrm{~mm}$ in all other patients and volunteers. In the patients, a contrast agent (gadopentetate dimeglumine [Magnevist; Bayer HealthCare Pharmaceuticals, Wayne, New Jersey] or gadodiamide [Omniscan; GE Healthcare, Piscataway, New Jersey]) was administered at $0.2 \mathrm{~mL}$ per kilogram of body weight.

BOLD fMRI was performed according to a previously published block design protocol in SG and WG tasks to evaluate language system. $^{25}$ This established presurgical language fMRI protocol was validated previously against the Wada test and intraoperative electrocorticography. ${ }^{25}$ There is also broad evidence that language fMRI is very sensitive and specific for language lateralization, even when using different paradigms and different experimental setups, as described in a recently published review. ${ }^{2}$ Therefore, it was deemed unnecessary to perform further invasive measures for this study, given the relevant risks for the patients. For the SG paradigm, patients were shown pictures with comic-like figures and were asked to repeat (silently, in their mind) a short standardized sentence that had been practiced with them before the scan (Fig 1). Twelve pictures existed, with 12 standard sentences. The duration of the stimulation phase in SGs was modulated on the achievement and cognitive ability of patients; 1 picture was shown for 2 seconds as a standard, but the presentation time was individually adapted ( 3 or 6 seconds) to compensate for tumor-related linguistic or other cognitive deficits, when required. ${ }^{32}$ In this way, it was feasible to use the task in all patients in the same way, including those with aphasic symptoms. In the WG paradigm, the patients were presented words of different categories and had to repeat as many items as possible, matching the category within 6 or 9 seconds (depending on cognitive ability). Both paradigms were practiced under supervision of the investigator before fMRI measurements and adapted, if required. The order of the 12 pictures (SG) or words (WG) was randomly chosen by computer and without direct repetitions (pseudorandomized) during the fMRI investigation. The BOLD fMRI protocol in both task types consisted of 1 offset $(9$ seconds for elimination of $\mathrm{T}^{\star}{ }^{\star}$-weighted effects), and 5 baseline phases (each phase lasting 18 seconds), alternating with 4 stimulation phases (each phase lasting 36 seconds). Therefore, each fMRI measurement lasted 243 seconds. Myopia and hyperopia could be corrected with special nonmagnetic mirror glasses, if needed. This protocol was established and developed in the University Hospital of Heidelberg Department of Neuroradiology. ${ }^{29}$

\section{Standardized Analysis of Individual fMRI Data}

Data processing software for fMRI was BrainVoyager (Brain Innovation B.V., Maastricht, Netherlands). Standardized processing included correction of motion artifacts, spatial-temporal smoothing, voxelwise linear correlation calculation for significant BOLD signal activation using an HRF, and correction for multiple comparisons. The HRF reflects delay of hemodynamic responses for detecting neuronal activity. As a result of the calculation, the correlation coefficient (" $r$ ") was achieved as a parameter for quality of BOLD fMRI signals, and the parameter " $\Delta S \%$ " was used as a measure of the relative BOLD-signal change within the activated BOLD cluster. Data processing was fully automated except for superimposition of conventional sequences on functional MR imaging. The tumor-affected structures of the brain could be determined in $\mathrm{T} 1^{\star}$-weighted 3D MR images by identification of anatomic landmarks. ${ }^{33,34}$ To detect these landmarks, coronal, sagittal, and transverse views of the 3D dataset were evaluated. Four ROIs were placed where $\mathrm{AMRI}$ analysis was to be
A

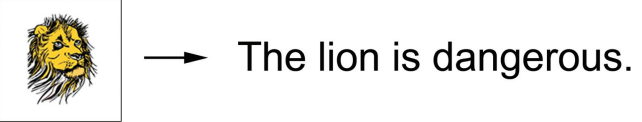

$\rightarrow$ Treateranatis heasy

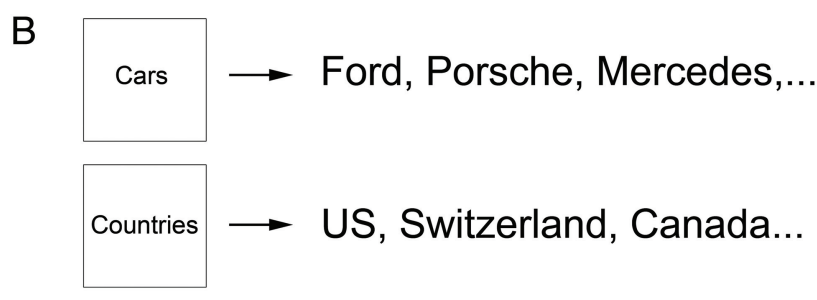

Fig 1. Applied visually triggered paradigms in clinical language fMRI. A, Two examples of the sentence generation paradigm are shown. Comic-like pictures such as the lion or the elephant are shown and the subject has to verbalize a standard simple sentence, such as "The lion is dangerous" or "The elephant is heavy." Altogether, this paradigm consists of a set of 12 visual comic-like stimuli, with 12 corresponding standard simple sentences. $B$, Two examples of the word-generation paradigm are shown. Generic terms like "cars" or "countries" are shown and the subject is asked to generate as many words as possible, such as, "Ford, Porsche, Mercedes," or "United States, Switzerland, Canada." Altogether, this paradigm consists of a set of 12 generic terms

performed: the Broca area in the left inferior frontal gyrus (Brodmann areas 44/45) and the Wernicke area in the left superior temporal gyrus (Brodmann area 22), with adjacent regions, including superior temporal sulcus, the middle temporal gyrus (Brodmann area 21, 22), the supramarginal gyrus (Brodmann area 40), and/or the angular gyrus (Brodmann area 39). In the right hemisphere, the ROIs were named $\mathrm{BR}$ and WR, according to their corresponding anatomic homologs. BOLD fMRI was analyzed individually after superimposing functional and 3D anatomic MR imaging for each patient and paradigm separately. The procedure was standardized as previously described in literature. ${ }^{25,29}$ The empirically minimal-proved cluster size of $36 \mathrm{~mm}^{3}$ was used as a standard. The cluster size of $36 \mathrm{~mm}^{3}$ has been our evaluation standard since 1997, and all reference data were established using this standard. This cluster size is small enough so as to not significantly obscure anatomic detail in the images, allowing the best possible determination of the exact anatomic correlate of the center of gravity of each activated BOLD cluster on 3D overlays of functional maps on structural images. On the other hand, the cluster size was large enough to reduce the number of very small activations (noise of single voxels) and was therefore a practical way of reducing falsepositives. At first, a very high statistical threshold value for the correlation coefficient $r$ to the applied HRF was chosen, resulting in no display of functional activity in the human brain (empty map). The initial maximum statistical threshold was set to $r=1.0$, resulting in an empty map, as only $100 \%$ perfect correlations of measured BOLDsignal time courses and applied hemodynamic reference functions would be displayed in the activation maps, which is never the case in a biologic system. From this maximum threshold, a continuous reduction was performed in steps of $r=0.02$. The statistical threshold value was reduced continuously until the activity with the highest correlation to HRF was displayed first. By further reducing the threshold value, the other activated areas were delineated in hierarchical order. This procedure was continued until activation in all previously defined ROIs (Broca, Wernicke, BR, WR) was identified, and thus all BOLD-signal time courses were analyzed individually under standardized conditions. As a lower limit, $r>0.4$ was defined for each 


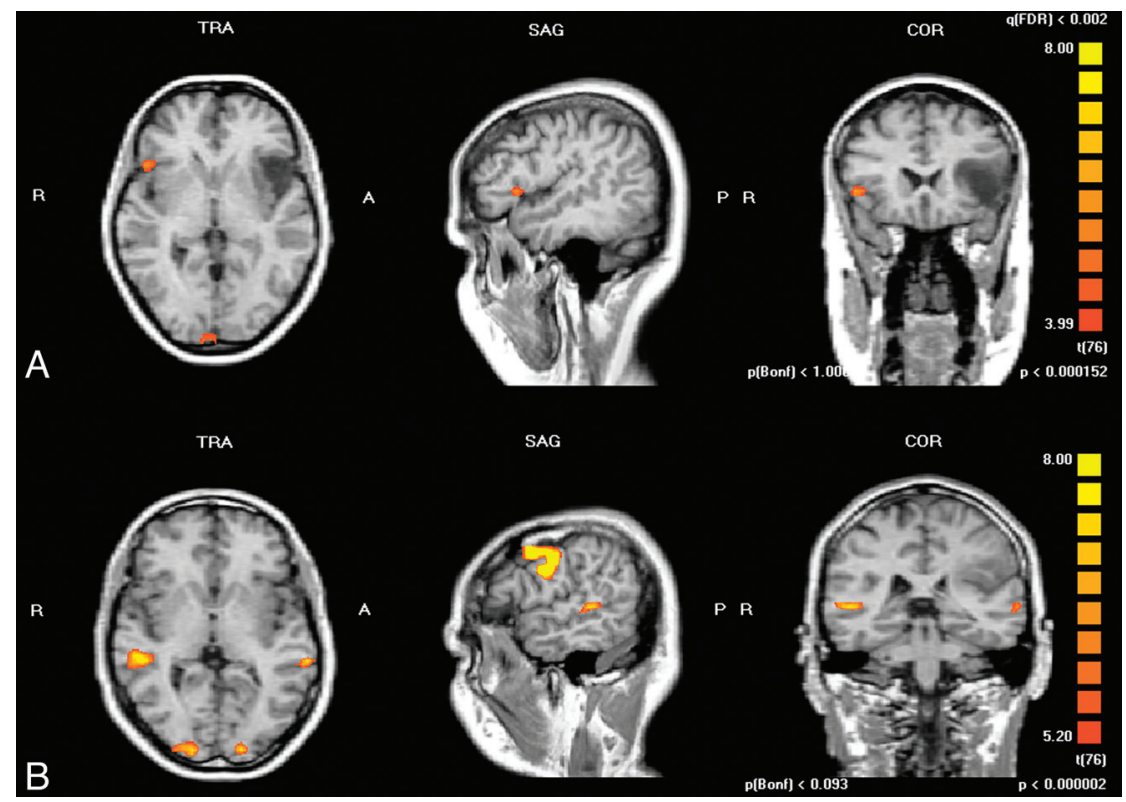

Fig 2. Representative instances of the patient group are demonstrated. $A, A$ right-handed 62-year-old patient with left hemispheric frontal astrocytoma (WHO II) is depicted. The word-generation paradigm was applied and a lateralization index of Broca area versus its right hemisphere homolog of -0.85 was calculated (lateralization toward right hemisphere). $B$, A right-handed 49-year-old patient with left hemispheric parietal astrocytoma (WHO II) is depicted. A sentence-generation paradigm was applied and a lateralization index of Wernicke area versus its right hemisphere homolog of -0.84 was calculated (lateralization toward right hemisphere).

region of interest. This was necessary to avoid mixing BOLD signals with background noise. If no BOLD signal in a region of interest was detected above this lower limit, it was defined as no activation. BOLD signals with a relative change $>5 \%$ were not included in the evaluation because such high-level activations most likely originate from draining veins rather than from capillaries.

To find the speech-dominant hemisphere, the local LI was calculated according to the established formula, LI = (left hemisphere-right hemisphere)/(-left hemisphere-right hemisphere), for Broca and Wernicke areas versus their right hemisphere homologs (Broca/BR and Wernicke/WR) separately. The values in the formula are number of activated voxels at the threshold of the peak activation of the nondominant hemisphere. ${ }^{35}$

\section{Statistical Analysis}

The Mann-Whitney $U$ test was used to analyze the statistical significance of differences in LI between patients and healthy volunteers separately for localization (Broca/BR and Wernicke/WR) and paradigm (SG and WG). Graphics were made as box-and-whisker plots. Statistical analysis was performed with SPSS software, version 11.0 (SPSS Inc., Chicago, Illinois).

\section{Results}

\section{Lateralization of Language in Broca Area}

Fig $2 A$ shows a case of a 62 -year-old patient with a left frontal astrocytoma (WHO grade II). The patient was right-handed. fMRI using the WG paradigm revealed language lateralization of the Broca area to the right side (LI Broca/BR $=-0.85$ ). No language or speech pathologies existed in this patient.

The WG task for Broca/BR revealed a median LI value of -0.215 , with a standard deviation of 0.768 . Mean value was 0.010. A significant difference in LI of Broca/BR between the 2 groups (healthy volunteers: median value, 0.970 ; standard deviation, 0.367 ; mean value 0.825$)$ could be detected $(P=.017)$ (Fig $3 A)$.
Performing SG in patients with brain tumor for Broca/BR, a median LI of 0.840 , with a standard deviation of 0.549 , was detected for the same paradigm. The mean value was 0.573 . In this paradigm, the lower LI in patients compared with healthy persons (median value, 0.860; standard deviation, 0.436 ; mean LI, 0.644) was not significant $(P=.851)$.

\section{Lateralization of Language in Wernicke Area}

Fig $2 B$ shows fMRI data of a 47-year-old right-handed patient with a left parietotemporal astrocytoma (WHO grade II). The patient suffered from focal seizures and anosmia. SG paradigm revealed only residual functional activity on the left side and substantial lateralization to the right anatomic homolog (LI Wernicke/WR $=-0.84$ ).

SG in the investigated patient group for Wernicke/WR revealed a calculated median value of 0.500 , with a standard deviation of 0.644 and a mean value of 0.232 . For this paradigm, a highly significant difference in the patient versus control group (median value, 0.960; standard deviation, 0.717; mean value, 0.653$)$ was found $(P=.007)$ (Fig $3 B)$. For WG, the LI in the patient group for Wernicke/WR had a median value of 0.475 , with a standard deviation of 0.592 . The mean value was 0.376 . A decrease in LI in the patient group compared with healthy volunteers (median value, 0.800; standard deviation, 0.547 ; mean value, 0.578$)$ was observed, but this reduction was not significant $(P=.310)$.

\section{Discussion}

Our results demonstrate robust feasibility of performing clinical language fMRI in patients with brain tumor, in particular for determining language lateralization through calculation of the local LI of Broca/BR and Wernicke/WR, respectively. Moreover, clinical fMRI provided evidence that patients with space-occupying brain masses in the language-dominant hemisphere show a significant amount of lateralization of 

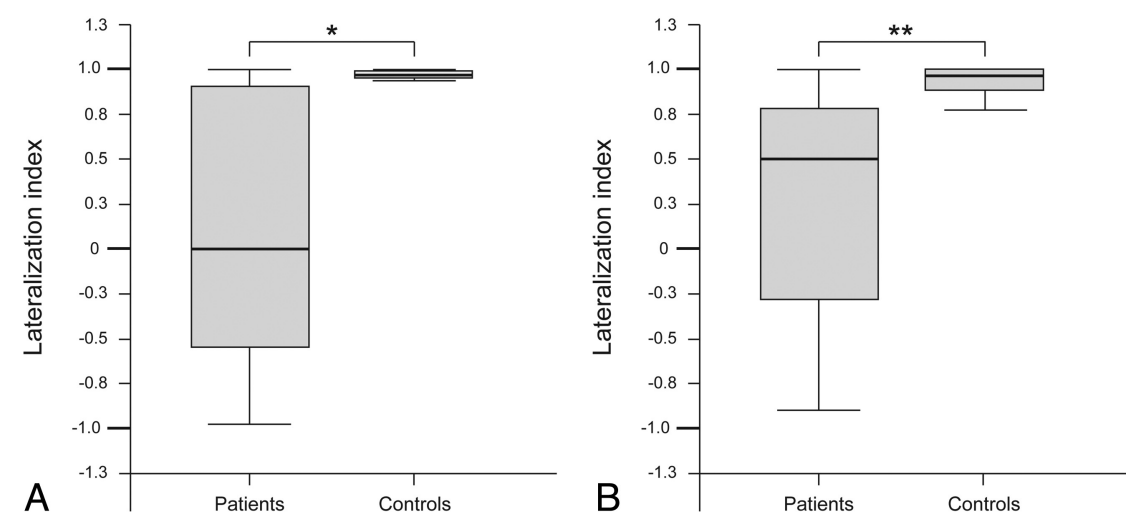

Fig 3. Local lateralization indexes in the patient group compared with healthy volunteers are depicted as box-and-whisker plots. $A$, $A$ significant decrease of the lateralization index in Broca area versus its right hemisphere homolog, when performing the word generation paradigm for brain tumors affecting Broca area, is shown $(P=.017)$. $B$, $A$ significant reduction of lateralization index in Wernicke area versus its right hemisphere homolog, when performing sentence generation paradigm for brain tumors affecting Wernicke area, is shown $(P=$ .007).

the Broca and Wernicke areas to the contralateral unaffected anatomic homologs of the corresponding language areas. In an fMRI and intraoperative electrical brain stimulation study with 77 patients with low-grade glioma, spatiotemporal functional reorganization was studied descriptively. ${ }^{22}$ The absence of presurgical aphasic symptoms was explained by compensation through perilesional brain areas rather than a shift of the dominant hemisphere to the contralateral site. These perilesional brain areas were partially infiltrated by the neoplasm but were nevertheless functionally active. fMRI has been used to show the compensation of language function of the left insula by adjacent brain areas. ${ }^{36} \mathrm{~A}$ longitudinal case study comparing pre- and postsurgical language lateralization in 2 patients demonstrated a shift of the dominant hemisphere to the opposite side after neurosurgery. ${ }^{37}$ In other studies using different imaging modalities, authors have assumed that cortical reorganization is a step-by-step process, with shortterm recruitment of perilesional areas and long-term recruitment of more distant areas within the dominant or contralateral hemisphere. ${ }^{2,38-40}$ Our study supports the hypothesis that cortical reorganization impacts language lateralization, with a possible shift toward the contralateral nondominant hemisphere.

Presurgical fMRI for language localization has been validated with former standard invasive methods like electrocorticography or Wada testing. ${ }^{5,8,41-44}$ Because of the lack of standardized experimental procedures, data analysis, and interpretation, the correlation of fMRI with standard methods was inconsistent in these studies. In our current trial, we used a previously validated and standardized protocol for performing the fMRI paradigms (WG and SG), MR data acquisition, data processing, and analysis. This method provided a technical success rate of $100 \%$ and a lateralization success rate of $98 \%$ for both paradigms. ${ }^{25}$ The use of multiple paradigms in clinical language fMRI is recommended to allow for different linguistic preferences and to increase the number of successful measurements and reproducibility of the fMRI results before brain surgery. A variety of different paradigms for performing clinical language fMRI are currently under investigation. ${ }^{45-47}$ Both paradigms applied in this study have been optimized and established in volunteers, have been in clinical use in a standardized way since 1998, and have been validated against invasive reference procedures (intraoperative electrocorticography and Wada testing), rendering further validations unnecessary. ${ }^{25,29}$ For this investigation, SG and WG paradigms were applied in a covert manner. This reduced motion, as well as magnetic, susceptibility artifacts (which are worse when performing overt tasks in clinical fMRI). ${ }^{48,49}$ Moreover, for determination of language lateralization, covert paradigms have proved better suitability, whereas overt paradigms are of limited diagnostic value for revealing the language-dominant hemisphere. ${ }^{31}$ Regarding this study in the Broca area, the WG paradigm led to the detection of a higher lateralization rate, whereas in the Wernicke area, SG resulted in a higher lateralization rate. The higher sensitivity for lateralization assessment of the Broca area by WG and Wernicke area by SG in our clinical fMRI protocol may be related to different linguistic content and semantic processing of the different paradigms. The fMRI results from SG and WG are confirmatory regarding language localization and synergistic regarding language lateralization. $^{31}$ Different methods for calculating LI have been used for determination of language dominance by fMRI. ${ }^{50}$ For this study, we used calculations of a regional LI for the region of interest pairs Broca/BR and Wernicke/WR to achieve reliable and more detailed information compared with a global LI calculation. Furthermore, for the determination of language dominance, it has been shown that regional LI correlates better with the results of Wada testing compared with global LI, which is based on voxel counts of the complete hemisphere. ${ }^{51}$

To date, literature regarding language lateralization in brain tumor patients is sparse. Different authors analyzed language lateralization after single or multiple brain resections. $^{22,23,52}$ Those studies led to the hypothesis that neuronal plasticity occurs predominantly in slow-growing neoplasms or after repetitive limited surgical resections. ${ }^{24,37}$ However, no study has been performed to evaluate language lateralization in a larger cohort of patients with brain tumor before neurosurgery. Taken together, our results indicate that brain tumors affecting the Broca or Wernicke areas have an impact on language lateralization. This might be part of a compensatory mechanism possibly allowing preservation of speech in these patients.

This noninvasively gained information adds to a purely clinical assessment of speech deficits in patients with brain 
tumor and is valuable in the diagnostic work-up. In addition, it has the potential to alter presurgical planning, as the extent of surgery may be adjusted to the amount of functional adaptations regarding eloquent brain areas. To prove this, further studies using intraoperative validation of neuroplastic changes, as indicated by fMRI, are warranted. Furthermore, these neuroplastic changes delineated by clinical language fMRI should be analyzed in terms of reorganization patterns offering therapeutic implications as well as prognostic information.

A limitation of this study was the heterogeneous patient population, which included different types of space-occupying masses. The rationale of this study was to assess the impact of intracranial space-occupying masses on language lateralization using standardized fMRI in a realistic clinical scenario. Therefore, the study population reflected a typical mixture of admissions to a major university hospital. Furthermore it should be considered that presurgical language fMRI is an evolving, though not yet clinically established, imaging approach. ${ }^{53}$ The primary aim of our study was to analyze a large number of patients with brain tumors to investigate language lateralization; therefore, we included all consecutive righthanded patients with left hemispheric brain tumors affecting the Broca and Wernicke areas, independent of the WHO grade. A cohort large enough to enable statistical comparisons between different entities of brain tumors would require a large multicenter trial.

In this study, we did not address local language reorganization in other functional brain areas surrounding the spaceoccupying masses or in secondary functional areas, which represents an additional limitation of our work. The rationale to set our focus on essential language centers and their anatomic homologs in the right hemisphere was driven by clinical relevance. The most important reason for presurgical language fMRI is to preserve essential language areas, namely, Broca and Wernicke areas, during surgery. Surgical damage to other language-related functional brain areas usually does not cause severe language deficits. ${ }^{54-56}$ Furthermore, it should be considered that the brain activation pattern is heavily dependent on the language paradigms applied and therefore differs dramatically when looking at cortical language networks as a whole. The clinical information that can be derived from differences in the brain activation pattern as a whole is still unclear; therefore, this information cannot be applied in a strict sense for presurgical fMRI. This topic may be addressed in future work on language network reorganization in the case of brain tumors.

\section{Conclusions}

Standardized clinical fMRI enabled robust localization of Broca and Wernicke language areas, and their anatomic homologs in the right hemisphere, in patients with brain tumors affecting those essential language centers. The intracranial space-occupying masses exhibited statistically significant effects on language lateralization by a shift of activation toward the unaffected right hemisphere. This may be related to compensatory mechanisms of the human brain and to maintaining communicative abilities.

\section{References}

1. Ojemann G, Ojemann J, Lettich E, et al. Cortical language localization in left, dominant hemisphere. An electrical stimulation mapping investigation in 117 patients. J Neurosurg 1989;71:316-26

2. Dym RJ, Burns J, Freeman K, et al. Is functional MR imaging assessment of hemispheric language dominance as good as the Wada test? A meta-analysis. Radiology 2011;261:446-55

3. Springer JA, Binder JR, Hammeke TA, et al. Language dominance in neurologically normal and epilepsy subjects: a functional MRI study. Brain 1999;122: 2033-46

4. Gartus A, Foki T, Geissler A, et al. Improvement of clinical language localization with an overt semantic and syntactic language functional MR imaging paradigm. AJNR Am J Neuroradiol 2009;30:1977-85

5. Binder JR, Swanson SJ, Hammeke TA, et al. Determination of language dominance using functional MRI: a comparison with the Wada test. Neurology 1996;46:978-84

6. Desmond JE, Sum JM, Wagner AD, et al. Functional MRI measurement of language lateralization in Wada-tested patients. Brain 1995;118:1411-19

7. Rutten GJ, van Rijen PC, van Veelen CW, et al. Language area localization with three-dimensional functional magnetic resonance imaging matches intrasulcal electrostimulation in Broca's area. Ann Neurol 1999;46:405-08

8. FitzGerald DB, Cosgrove GR, Ronner S, et al. Location of language in the cortex: a comparison between functional MR imaging and electrocortical stimulation. AJNR Am J Neuroradiol 1997;18:1529-39

9. Cousin E, Baciu M, Pichat C, et al. Functional MRI evidence for language plasticity in adult epileptic patients: preliminary results. Neuropsychiatr Dis Treat 2008;4:235-46

10. Mbwana J, Berl MM, Ritzl EK, et al. Limitations to plasticity of language network reorganization in localization related epilepsy. Brain 2009;132:347-56

11. Rosenberger LR, Zeck J, Berl MM, et al. Interhemispheric and intrahemispheric language reorganization in complex partial epilepsy. Neurology 2009; 72:1830-36

12. Cao Y, Vikingstad EM, George KP, et al. Cortical language activation in stroke patients recovering from aphasia with functional MRI. Stroke 1999;30: 2331-40

13. Rosen HJ, Petersen SE, Linenweber MR, et al. Neural correlates of recovery from aphasia after damage to left inferior frontal cortex. Neurology 2000;55: 1883-94

14. Saur D, Lange R, Baumgaertner A, et al. Dynamics of language reorganization after stroke. Brain 2006;129:1371-84

15. Heiss WD. WSO Leadership in Stroke Medicine Award Lecture Vienna, September 26, 2008: functional imaging correlates to disturbance and recovery of language function. Int J Stroke 2009;4:129-36

16. Hess G, Donoghue JP. Long-term depression of horizontal connections in rat motor cortex. Eur J Neurosci 1996;8:658-65

17. Hess G, Aizenman CD, Donoghue JP. Conditions for the induction of longterm potentiation in layer II/III horizontal connections of the rat motor cortex. J Neurophysiol 1996;75:1765-78

18. Fandino J, Kollias SS, Wieser HG, et al. Intraoperative validation of functional magnetic resonance imaging and cortical reorganization patterns in patients with brain tumors involving the primary motor cortex. J Neurosurg 1999;91: $238-50$

19. Seitz RJ, Huang Y, Knorr U, et al. Large-scale plasticity of the human motor cortex. Neuroreport 1995;6:742-44

20. Atlas SW, Howard RS 2nd, Maldjian J, et al. Functional magnetic resonance imaging of regional brain activity in patients with intracerebral gliomas: findings and implications for clinical management. Neurosurgery 1996;38:329-38

21. Mueller WM, Yetkin FZ, Hammeke TA, et al. Functional magnetic resonance imaging mapping of the motor cortex in patients with cerebral tumors. Neurosurgery 1996;39:515-20; discussion 520-21

22. Duffau H, Capelle L, Denvil D, et al. Functional recovery after surgical resection of low grade gliomas in eloquent brain: hypothesis of brain compensation. J Neurol Neurosurg Psychiatry 2003;74:901-07

23. Duffau H, Denvil D, Capelle L. Long term reshaping of language, sensory, and motor maps after glioma resection: a new parameter to integrate in the surgical strategy. J Neurol Neurosurg Psychiatry 2002;72:511-16

24. Desmurget $\mathrm{M}$, Bonnetblanc F, Duffau H. Contrasting acute and slow-growing lesions: a new door to brain plasticity. Brain 2007;130:898-914

25. Stippich C, Rapps N, Dreyhaupt J, et al. Localizing and lateralizing language in patients with brain tumors: feasibility of routine preoperative functional MR imaging in 81 consecutive patients. Radiology 2007;243:828-36

26. Fernandez B, Cardebat D, Demonet JF, et al. Functional MRI follow-up study of language processes in healthy subjects and during recovery in a case of aphasia. Stroke 2004;35:2171-76

27. Weber B, Wellmer J, Schur S, et al. Presurgical language fMRI in patients with drug-resistant epilepsy: effects of task performance. Epilepsia 2006;47:880-86

28. Niskanen E, Kononen M, Villberg V, et al. The effect of fMRI task combinations on determining the hemispheric dominance of language functions. Neuroradiology 2012;54:393-405

29. Stippich C, Mohammed J, Kress B, et al. Robust localization and lateralization 
of human language function: an optimized clinical functional magnetic resonance imaging protocol. Neurosci Lett 2003;346:109-13

30. Annett M. A classification of hand preference by association analysis. $\mathrm{Br} \mathrm{J}$ Psychol 1970;61:303-21

31. Partovi S, Konrad F, Karimi S, et al. Effects of covert and overt paradigms in clinical language fMRI. Acad Radiol 2012;19:518-25

32. Konrad F, Nennig E, Ochmann H, et al. [Does the individual adaptation of standardized speech paradigms for clinical functional magnetic resonance imaging (fMRI) affect the localization of the language-dominant hemisphere and of Broca's and Wernicke's areas]. [Article in German] Rofo 2005;177: 381-85

33. Naidich TP, Valavanis AG, Kubik S. Anatomic relationships along the lowmiddle convexity: part I-normal specimens and magnetic resonance imaging. Neurosurgery 1995;36:517-32

34. Naidich TP, Hof PR, Gannon PJ, et al. Anatomic substrates of language: emphasizing speech. Neuroimaging Clin N Am 2001;11:305-41, ix

35. Szaflarski JP, Binder JR, Possing ET, et al. Language lateralization in lefthanded and ambidextrous people: fMRI data. Neurology 2002;59:238-44

36. Duffau H, Bauchet L, Lehericy S, et al. Functional compensation of the left dominant insula for language. Neuroreport 2001;12:2159-63

37. Pillai JJ. Insights into adult postlesional language cortical plasticity provided by cerebral blood oxygen level-dependent functional MR imaging. AJNR AmJ Neuroradiol 2010;31:990-96

38. Weiller C, Isensee C, Rijntjes M, et al. Recovery from Wernicke's aphasia: a positron emission tomographic study. Ann Neurol 1995;37:723-32

39. Musso M, Weiller C, Kiebel S, et al. Training-induced brain plasticity in aphasia. Brain 1999;122:1781-90

40. Thiel A, Herholz K, Koyuncu A, et al. Plasticity of language networks in patients with brain tumors: a positron emission tomography activation study. Ann Neurol 2001;50:620-29

41. Woermann FG, Jokeit H, Luerding $\mathrm{R}$, et al. Language lateralization by Wada test and fMRI in 100 patients with epilepsy. Neurology 2003;61:699-701

42. Pouratian N, Bookheimer SY, Rex DE, et al. Utility of preoperative functional magnetic resonance imaging for identifying language cortices in patients with vascular malformations. J Neurosurg 2002;97:21-32

43. Yetkin FZ, Mueller WM, Morris GL, et al. Functional MR activation correlated with intraoperative cortical mapping. AJNR Am J Neuroradiol 1997;18: 1311-15

44. Yetkin FZ, Swanson S, Fischer M, et al. Functional MR of frontal lobe activation: comparison with Wada language results. AJNR Am J Neuroradiol 1998;19:1095-98

45. Mahdavi A, Houshmand S, Oghabian MA, et al. Developing optimized fMRI protocol for clinical use: comparison of different language paradigms. J Magn Reson Imaging 2011;34:413-19

46. Gutbrod K, Spring D, Degonda N, et al. Determination of language dominance: Wada test and fMRI compared using a novel sentence task. J Neuroimaging 2012;22:266-74

47. Smits M, Visch-Brink E, Schraa-Tam CK, et al. Functional MR imaging of language processing: an overview of easy-to-implement paradigms for patient care and clinical research. Radiographics 2006;26:S145-58

48. Barch DM, Sabb FW, Carter CS, et al. Overt verbal responding during fMRI scanning: empirical investigations of problems and potential solutions. $\mathrm{Neu}$ roimage 1999;10:642-57

49. Birn RM, Bandettini PA, Cox RW, et al. Event-related fMRI of tasks involving brief motion. Hum Brain Mapp 1999;7:106-14

50. Jansen A, Menke R, Sommer J, et al. The assessment of hemispheric lateralization in functional MRI-robustness and reproducibility. Neuroimage 2006;33: 204-17

51. Spreer J, Arnold S, Quiske A, et al. Determination of hemisphere dominance for language: comparison of frontal and temporal fMRI activation with intracarotid amytal testing. Neuroradiology 2002;44:467-74

52. $\mathrm{Wu} \mathrm{CX}, \mathrm{Pu} \mathrm{S}, \mathrm{Lin} \mathrm{Y}$, et al. Fractionated resection on low grade gliomas involving Broca's area and insights to brain plasticity. Chin Med J (Engl) 2008;121: 2026-30

53. Tieleman A, Deblaere K, Van Roost D, et al. Preoperative fMRI in tumour surgery. Eur Radiol 2009;19:2523-34

54. Roth HL. Finding language in the matter of the brain: origins of the clinical aphasia examination. Semin Neurol 2002;22:335-48

55. Binder JR, Frost JA, Hammeke TA, et al. Human brain language areas identified by functional magnetic resonance imaging. J Neurosci 1997;17:353-62

56. Engström M, Ragnehed M, Lundberg P, et al. Paradigm design of sensorymotor and language tests in clinical fMRI. Neurophysiol Clin 2004;34: 267-77 